\title{
Tube Voltage, DNA Double-Strand Breaks, and Image Quality in Coronary CT Angiography
}

\author{
Zhu Xiao Lin, $\mathrm{MSc}^{1,2 *}$, Fan Zhou, MSc ${ }^{1 *}$, U. Joseph Schoepf, $\mathrm{MD}^{1,3}$, Balakrishnan Pillai, BS ${ }^{3}$, \\ Chang Sheng Zhou, $\mathrm{MSc}^{1}$, Wei Quan, $\mathrm{MSc}^{1}$, Xue Qin Bao, $\mathrm{BS}^{1}$, Guang Ming Lu, MD', \\ Long Jiang Zhang, MD, PhD ${ }^{1}$
}

${ }^{1}$ Department of Medical Imaging, Jinling Hospital, Medical School of Nanjing University, Nanjing, China; ${ }^{2}$ Department of Medical Imaging, Yantaishan Hospital, Yantai, China; ${ }^{3}$ Division of Cardiovascular Imaging, Department of Radiology and Radiological Science, Medical University of South Carolina, Charleston, SC, USA

Objective: To evaluate the effects of tube voltage on image quality in coronary CT angiography (CCTA), the estimated radiation dose, and DNA double-strand breaks (DSBs) in peripheral blood lymphocytes to optimize the use of CCTA in the era of low radiation doses.

Materials and Methods: This study included 240 patients who were divided into 2 groups according to the DNA DSB analysis methods, i.e., immunofluorescence microscopy and flow cytometry. Each group was subdivided into 4 subgroups: those receiving CCTA only with different tube voltages of 120, 100, 80, or $70 \mathrm{kVp}$. Objective and subjective image quality was evaluated by analysis of variance. Radiation dosages were also recorded and compared.

Results: There was no significant difference in demographic characteristics between the 2 groups and 4 subgroups in each group (all $p>0.05$ ). As tube voltage decreased, both image quality and radiation dose decreased gradually and significantly. After CCTA, $\gamma-\mathrm{H} 2 \mathrm{AX}$ foci and mean fluorescence intensity in the 120-, 100-, 80-, and 70-kVp groups increased by $0.14,0.09$, 0.07 , and 0.06 foci per cell and 21.26, 9.13, 8.10, and 7.13 (all $p<0.05$ ), respectively. The increase in the DNA DSB level in the 120-kVp group was higher than those in the other 3 groups (all $p<0.05$ ), while there was no significant difference in the DSBs levels among these latter groups (all $p>0.05$ ).

Conclusion: The 100-kVp tube voltage may be optimal for CCTA when weighing DNA DSBs against the estimated radiation dose and image quality, with further reductions in tube voltage being unnecessary for CCTA.

Keywords: Radiation dosage; Computed tomography angiography; Immunofluorescence microscopy; Flow cytometry;

DNA damage

\section{INTRODUCTION}

Coronary CT angiography (CCTA) has been widely used for diagnosing coronary artery disease, but the radiation dose in CCTA should not be underestimated (1-3). At present, various techniques for reducing radiation dose have been developed, such as prospective electrocardiogram (ECG) triggering, reduction in tube voltage, and iterative reconstruction
(IR) (4-10). Since the radiation dose is proportional to the square of the tube voltage, reducing tube voltage is the most effective method to reduce radiation dose. A tube voltage of $70 \mathrm{kVp}$ in CCTA has been reported to be feasible for obtaining high diagnostic accuracy (10); however, this approach is mostly restricted to subjects with a body mass index (BMI) less than $25 \mathrm{~kg} / \mathrm{m}^{2}$ to ensure diagnostic image quality. A reduction in the tube voltage can result in

Received: December 24, 2019 Revised: February 16, 2020 Accepted: March 5, 2020

This work was supported by The National Key Research and Development Program of China (2017YFC0113400 for L.J.Z.).

*These authors contributed equally to this work.

Corresponding author: Long Jiang Zhang, MD, PhD, Department of Medical Imaging, Jinling Hospital, Medical School of Nanjing University, Nanjing 210002, China.

- Tel: (86) 013405833176 • Fax: (86) 02580860185 • E-mail: kevinzhlj@163.com

This is an 0pen Access article distributed under the terms of the Creative Commons Attribution Non-Commercial License (https:// creativecommons.org/licenses/by-nc/4.0) which permits unrestricted non-commercial use, distribution, and reproduction in any medium, provided the original work is properly cited. 
deterioration of image quality and subsequent limitations in clinical use, and even an increase in the scattered X-rays absorbed by the subjects. Thus, although minimizing radiation dosage is an overarching goal in any form of diagnostic radiology, in the realm of CCTA, it can eventually result in loss of clinical utility, and hence it is unreasonable to pursue the lowest estimated radiation dose in every case.

In clinical practice, physical measurements such as effective dose, volumetric CT dose index (CTDIvol), and dose-length product (DLP) have been routinely used to describe the radiation doses received by subjects, but these metrics cannot reflect the interaction of $X$-rays with human tissue. Unlike these parameters, biological indicators could be more effective in assessing the true effect of ionizing radiation on human tissue. DNA double-strand breaks (DSBs) have been shown to be the most severe form of cellular damage produced by radiation damage (11). Immunofluorescence microscopy using probes against $\gamma-\mathrm{H} 2 \mathrm{AX}$, a marker of DNA DSBs, has been used to detect DSBs with much higher sensitivity than cytogenetic analysis of dicentric chromosomes (1 mGy vs. $100 \mathrm{mGy}$ ) (12-15). Furthermore, analysis of the mean fluorescence intensities (MFIs) of $\gamma$-H2AX using flow cytometry has been shown to be more objective and was used in many studies (16-18). In addition to $\gamma-\mathrm{H} 2 \mathrm{AX}$, other markers of DNA damage such as ataxia telangiectasia-mutated protein and P53 protein (19) can be used to detect DNA damage, but these markers are not used widely.

Biological effects have not been adequately analyzed in the assessment of radiation reduction strategies currently employed in clinical practice. The first study to assess the influence of CCTA scanning parameters on the biological effects of X-rays was published in 2010 , which found that the DSB levels in patients examined with $100 \mathrm{kVp}$ were approximately $30 \%$ lower than in patients examined with $120 \mathrm{kVp}$ (20). However, another study reported (21) that lower tube voltage could increase DNA DSBs when the tube current is elevated. To the best of our knowledge, no study has yet attempted to optimize tube voltage and image quality from the perspective of the biological effects in CCTA studies.

Thus, the purpose of this study was to evaluate the effects of tube voltage on the image quality of CCTA, estimated radiation dose, and DSBs in peripheral blood lymphocytes, in order to optimize the use of CCTA in the era of low radiation doses.

\section{MATERIALS AND METHODS}

\section{Patients}

The study was approved by the local ethics committee, and written informed patient consent was obtained from each subject. Altogether 240 patients with suspected coronary artery disease were included in the study. The enrollment criteria were as follows: age greater than 18 years, BMI less than $25 \mathrm{~kg} / \mathrm{m}^{2}$, absence of lymphoma or leukemia, no radiation therapy or chemotherapy within 6 months prior to this study, no examinations using X-rays or nuclear medicine studies within 1 week prior to this study, no contraindications to iodinated contrast agents (such as hyperthyroidism and heart failure), and no history of coronary artery bypass grafting or coronary artery stent implantation.

The patients were divided into four groups based on tube voltages used during CCTA, i.e., $120 \mathrm{kVp}$ (A group), $100 \mathrm{kVp}$ (B group), $80 \mathrm{kVp}$ (C group), and $70 \mathrm{kVp}$ and (D group). Each group was further divided into 2 subgroups by random number generation. Patients in group A1-D1 underwent immunofluorescence microscopy for DNA DSB analysis, while the patients in Group A2-D2 underwent flow cytometric analysis. Except for different tube voltages, other scanning parameters were kept the same for the patients in all subgroups.

\section{CCTA Scan and Estimation of Radiation Dose \\ CCTA examination was performed in end-inspiratory} breath-hold with heart rate monitoring by a prospective ECG-triggered sequence on a second-generation dualsource CT scanner (SOMATOM ${ }^{\circledR}$ Definition Flash, Siemens Healthineers, Forchheim, Germany) after intravenous administration of $60 \mathrm{~mL}$ of iopromide (Ultravist ${ }^{\circledR}$, Bayer AG, Berlin, Germany) with a concentration of $370 \mathrm{mg}$ of iodine $/ \mathrm{mL}$ at $5 \mathrm{~mL} / \mathrm{s}$. Automated bolus triggering was used to determine the delay time of the arterial phase. The area of interest was placed in the aorta with an automated trigger threshold of 100 Hounsfield units (HU). Tube voltages were $120,100,80$, and $70 \mathrm{kVp}$, respectively. The remaining scanning parameters were consistent: effective tube current, $280 \mathrm{mAs}$; rotation time $=0.28$ seconds; collimation $=0.6 \mathrm{~mm} \times 128$. The scanning range was from the tracheal carina to the bottom of the heart. Sinogram affirmed iterative reconstruction (SAFIRE; Siemens Healthineers, Forchheim, Germany, strength level of 3) was used per default. Raw data were transferred to a clinical 
workstation for image postprocessing.

The CTDIvol and DLP were recorded for each CCTA study according to the patient's study protocol report. Effective dose was estimated by multiplying the DLP by a conversion factor of $0.014 \mathrm{mSv} / \mathrm{mGy} \cdot \mathrm{cm}(22)$.

\section{Blood Sampling}

Two milliliters of whole venous blood were taken from the antecubital vein before and 20 minutes after the examination, respectively. After dilution with phosphatebuffered saline (PBS), peripheral blood mononuclear cells (PBMCs) were obtained by Ficoll density-gradient centrifugation and the samples were washed twice with PBS.

\section{Immunocytochemical Staining and Fluorescence Microscopy}

The lymphocytes of groups A1, B1, C1, and D1 were resuspended in $2 \mathrm{~mL}$ of 1640 medium. Then, 30- $\mu \mathrm{L}$ samples were placed on each cover glass and incubated at room temperature for about 1 hour. The cells were then fixed with $200 \mu \mathrm{L}$ of $4 \%$ paraformaldehyde for 20 minutes, and permeabilized with $0.3 \%$ Triton X-100 PBS for 10 minutes at room temperature. After being blocked with sheep serum albumin for 30 minutes at room temperature, the samples were incubated with rabbit anti- $\gamma \mathrm{H} 2 \mathrm{AX}$ antibody (clone JBW301, Upstate, Chandlers Ford, UK) overnight and with mouse anti-rabbit secondary antibodies (Invitrogen, Paisley, UK) for 1 hour. Finally, the samples were stained using UltraCruzTM (Santa Cruz, CA, USA, containing 4, 6-diamidino-2-phenylindole) and mounted with neutral resin. Fluorescence images were obtained by using an Olympus IX71 inverted fluorescence microscope (Olympus Co., Tokyo, Japan). For the quantitative analysis, red fluorescent foci (Supplementary Fig. 1) were counted in 200 cells to account via background correction. The number of foci in 200 cells was measured and averaged. Analysis of foci in PBMCs was performed in only those cells with lymphocyte-like morphology. The number of $X$-ray-induced $\gamma-\mathrm{H} 2 \mathrm{AX}$ foci was obtained by subtracting the number of $\gamma-\mathrm{H} 2 \mathrm{AX}$ foci of the samples obtained just before and 20 minutes after the CCTA.

\section{Flow Cytometry and Mean of Fluorescence Intensity}

The lymphocytes in groups A2, B2, C2, and D2 were diluted to $1 \times 10^{6}$ cells $/ \mathrm{mL}$ with 1640 medium, fixed with $4 \%$ paraformaldehyde at room temperature for 15 minutes, and permeabilized with $0.1 \%$ Triton X-100 for 10 minutes.
The samples were washed with PBS 3 times after each step. Samples were incubated with FITC-CD3 antibody (BD Biosciences, Franklin Lakes, NJ, USA) and APC $-\gamma-\mathrm{H} 2 \mathrm{AX}$ antibody (BD Biosciences) for 30 minutes protected from light. The samples were then analyzed by a flow cytometer (BD Calibur, BD Bioscience). Based on forward and side light scattering, PBMCs were gated for viable single-cell events. MFI of $\gamma$-H2AX in positive T-lymphocytes was evaluated using FlowJo software (V10.0.2, Tree Star, Inc., Ashland, OR, USA) (Supplementary Fig. 2).

\section{Objective and Subjective Image Quality Evaluations}

Image quality was objectively evaluated by measuring the average CT value in $\mathrm{HU}$ and image noise by the standard deviation (SD) in circular regions of interest (ROIs). These measurements were performed in the right coronary artery (RCA), left anterior descending artery (LAD), left circumflex artery (LCX), aorta, and sternocleidomastoid muscle. The ROIs were either circular or ellipsoidal and covered the maximum region of the vessel, excluding walls, plaques, and calcium regions. ROIs within coronary arteries and muscle tissue had an area of $5-10 \mathrm{~mm}^{2}$, while ROIs within the aorta were $10 \mathrm{~cm}^{2}$ in area (Supplementary Fig. 3). The signal-to-noise ratio (SNR) was calculated as the ratio between the mean $C T$ value and the $\mathrm{SD}$, while contrast-to-noise ratio (CNR) was calculated as (mean CT value $_{\text {organ of interest }}$ - mean $\mathrm{CT}$ value muscle $_{\text {) }} / \mathrm{SD}_{\text {heart muscle }}$.

Two independent blinded observers (with five- and threeyear experience in CCTA, respectively) scored the overall image quality using a 5-point scale (Supplementary Fig. 4), where 5 = excellent; $4=$ above average; $3=$ acceptable; $2=$ suboptimal, but images are still interpretable; and $1=$ very poor (nondiagnostic). Image noise was assessed as follows: $5=$ minimal noise; 4 = less than average; 3 = average; 2 = higher than average; $1=$ unacceptably high (23). In case of disagreement between the two observers, consensus was reached in a joint reading to determine the final image quality score.

\section{Statistical Analysis}

Data in this study were analyzed using Statistical Product and Service Solutions (SPSS) software (version 19, IBM Corp., Armonk, NY, USA). Shapiro-Wilk testing was applied to analyze normal distribution of data sets. Normally distributed variables were expressed as mean \pm SD values, and non-normally distributed variables were displayed as median with interquartile ranges. Sex was compared by 
chi-squared test, and other demographic characteristics and radiation dose between groups were compared using independent sample $t$ test and one-way analysis of variance, if appropriate. Wilcoxon signed-rank test was used for DNA DSBs before and after CCTA, and Kruskal-Wallis rank sum test was used for comparison of $\gamma$-H2AX foci and MFI among groups. Analysis of variance was performed to compare the objective measurements (CT value, SNR, and CNR) of the standard in four subgroups. Interobserver variability between the 2 observers with regard to subjective image quality assessment was evaluated with $\kappa$ statistics $(\kappa<$ 0.20 , poor agreement; $0.21<\kappa<0.40$, fair agreement; 0.41 $<\kappa<0.60$, moderate agreement; $0.61<\kappa<0.80$, good agreement; $0.81<\kappa<1.00$, very good agreement). $P<0.05$ was considered statistically significant.

\section{RESULTS}

\section{Demographic Characteristics and Radiation Dose}

The distribution of demographic data among groups is shown in Table 1. There were no significant differences in demographic characteristics among the four subgroups (subgroup A1, B1, C1, D1 and subgroup A2, B2, C2, D2) (all $p>0.05)$. As the tube voltage decreased, the estimated radiation dose (effective dose, CTDIvol, and DLP) decreased as well (all $p<0.001$ ) (Fig. 1). Table 2 shows the radiation doses in different tube voltage groups.

\section{$\gamma-\mathrm{H} 2 \mathrm{AX}$ Yield after CCTA}

\section{$\gamma-H 2 A X$ Foci Level}

The median baseline levels of $\gamma-\mathrm{H} 2 \mathrm{AX}$ foci were 0.24 ( 0.16 , $0.33)$ foci/cell in subgroup $A 1,0.20(0.18,0.23)$ foci/cell in subgroup B1, $0.28(0.18,0.23)$ foci/cell in subgroup C1, and $0.21(0.17,0.27)$ foci/cell in subgroup D1. There was no significant difference in the baseline levels of $\gamma-\mathrm{H} 2 \mathrm{AX}$

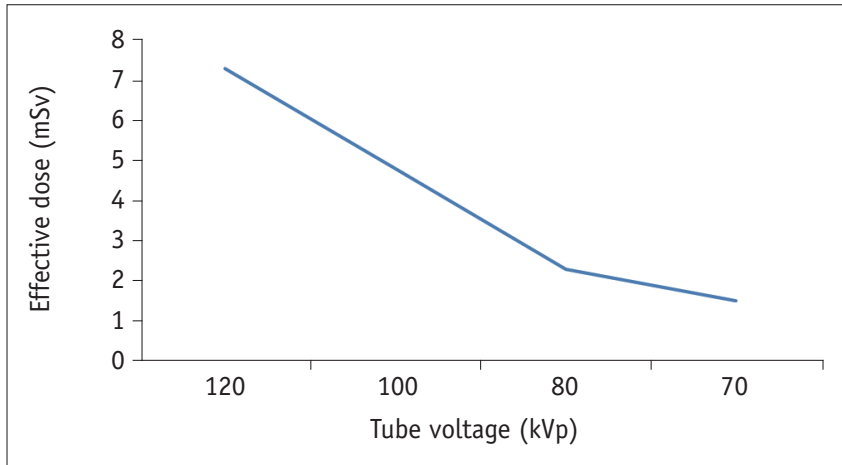

A
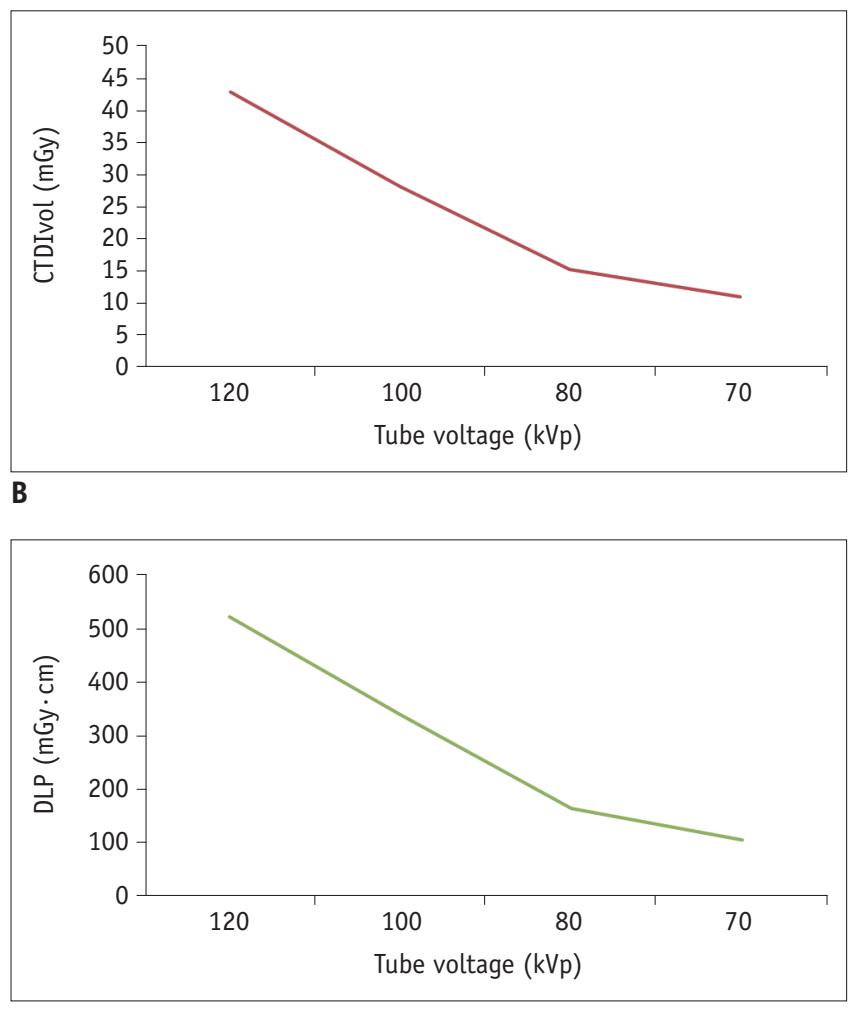

Fig. 1. Estimated radiation dose trends over different tube voltages.

As tube voltage decreases, estimated radiation dose (effective dose (A), CTDIvol (B), and DLP (C)) decreases. CTDIvol = volumetric CT dose index, DLP $=$ dose-length product

Table 1. Demographic Characteristics and Estimated Radiation Doses among Subgroups

\begin{tabular}{|c|c|c|c|c|c|c|c|c|c|c|}
\hline \multirow{2}{*}{ Parameters } & \multicolumn{4}{|c|}{ Immunofluorescence Staining } & \multicolumn{6}{|c|}{ Flow Cytometry } \\
\hline & A1 & B1 & $\mathrm{C} 1$ & D1 & $P$ & $\mathrm{~A} 2$ & B2 & $\mathrm{C2}$ & D2 & $P$ \\
\hline Sex, number & & & & & 0.199 & & & & & 0.750 \\
\hline Male & 22 & 19 & 19 & 14 & & 16 & 19 & 16 & 15 & \\
\hline Female & 8 & 11 & 11 & 16 & & 14 & 11 & 14 & 15 & \\
\hline Age, years & $53 \pm 13$ & $56 \pm 12$ & $56 \pm 12$ & $54 \pm 12$ & 0.243 & $56 \pm 10$ & $59 \pm 11$ & $59 \pm 11$ & $55 \pm 11$ & 0.320 \\
\hline Height, $\mathrm{cm}$ & $169.5 \pm 5.7$ & $167.6 \pm 8.9$ & $169.3 \pm 6.2$ & $167.2 \pm 6.8$ & 0.453 & $167.0 \pm 7.2$ & $167.3 \pm 8.7$ & $167.0 \pm 7.0$ & $166.5 \pm 8.0$ & 0.985 \\
\hline Weight, kg & $68.4 \pm 6.5$ & $66.5 \pm 9.3$ & $69.3 \pm 5.2$ & $66.8 \pm 6.8$ & 0.363 & $66.1 \pm 7.6$ & $66.9 \pm 8.3$ & $66.6 \pm 6.4$ & $64.1 \pm 13.1$ & 0.623 \\
\hline BMI, $\mathrm{kg} / \mathrm{m}^{2}$ & $23.7 \pm 1.1$ & $23.5 \pm 1.5$ & $24.2 \pm 0.8$ & $23.8 \pm 1.0$ & 0.189 & $23.6 \pm 1.2$ & $23.8 \pm 1.0$ & $23.8 \pm 0.9$ & $23.0 \pm 3.8$ & 0.363 \\
\hline
\end{tabular}

$A=120 \mathrm{kVp}$ group, $B=100 \mathrm{kVp}$ group, $B M I=$ body mass index, $C=80 \mathrm{kVp}$ group, $D=70 \mathrm{kVp}$ group 
Table 2. Radiation Doses at Different Tube Voltages

\begin{tabular}{lccc}
\hline \multicolumn{1}{c}{ Groups } & $\begin{array}{c}\text { Effective dose, } \\
\mathrm{mSv}\end{array}$ & $\begin{array}{c}\text { CTDIvol, } \\
\mathrm{mGy}\end{array}$ & $\begin{array}{c}\mathrm{DLP}, \\
\mathrm{mGy} \cdot \mathrm{cm}\end{array}$ \\
\hline $120 \mathrm{kVp}(\mathrm{n}=60)$ & $7.3 \pm 1.8$ & $42.9 \pm 7.7$ & $523.3 \pm 130.1$ \\
$100 \mathrm{kVp}(\mathrm{n}=60)$ & $4.8 \pm 0.7$ & $28.2 \pm 4.4$ & $339.7 \pm 47.2$ \\
$80 \mathrm{kVp}(\mathrm{n}=60)$ & $2.3 \pm 0.4$ & $15.3 \pm 2.8$ & $163.9 \pm 27.9$ \\
$70 \mathrm{kVp}(\mathrm{n}=60)$ & $1.5 \pm 0.2$ & $11.0 \pm 2.7$ & $105.0 \pm 16.7$ \\
$p$ value & $<0.001$ & $<0.001$ & $<0.001$ \\
\hline
\end{tabular}

Values are mean \pm standard deviation. CTDIvol = volumetric CT dose index, DLP = dose-length product

foci among the four subgroups $(p=0.066)$. However, $\gamma-\mathrm{H} 2 \mathrm{AX}$ foci in the four subgroups increased significantly by $0.14,0.09,0.07$, and 0.06 foci/cell after CCTA (all $p<0.05$ ) in comparison with the baseline (Fig. 2).

\section{MFI of $\gamma-H 2 A X$}

The median baseline levels of $\gamma$-H2AX MFI were 71.1 (56.3, $129.0)$ in subgroup $A 2,83.2(67.7,135.0)$ in subgroup B2, $93.2(65.8,121.1)$ in subgroup $C 2$, and $88.2(68.5,235.2)$ in subgroup D2. There was no significant difference in the baseline levels of $\gamma$-H2AX MFI among the four subgroups ( $p=$ 0.196). However, radiation-induced $\gamma$-H2AX MFI increased significantly by $21.26,9.13,8.10,7.13$ after CCTA in all four subgroups (all $p<0.05$ ) (Fig. 3).

\section{Comparisons among Groups}

\section{$\gamma$-H2AX Foci Level}

Figure $4 \mathrm{~A}$ shows the changes in $\gamma-\mathrm{H} 2 \mathrm{AX}$ foci levels after CCTA in subgroups $A 1, B 1, C 1$, and D1, respectively. As tube voltage decreased, the increment in $\gamma-\mathrm{H} 2 \mathrm{AX}$ foci decreased gradually $(p=0.001)$. Post-hoc analysis showed that the change in $\gamma-\mathrm{H} 2 \mathrm{AX}$ foci levels in subgroup $\mathrm{A} 1$ was higher than those in the other three subgroups (B1, C1, D1) after CCTA ( $p=0.003, p=0.006, p<0.001$, respectively), but significant differences were not observed among the three subgroups $(p=0.184)$.

\section{MFI of $\gamma-H 2 A X$}

Figure 4B shows the changes in $\gamma$-H2AX MFI levels after CCTA in subgroups $A 2, B 2, C 2$, and D2. These findings corroborated those found via immunofluorescence microscopy. Namely, as tube voltage decreased, the increment in $\gamma$-H2AX MFI decreased gradually $(p<0.001)$, but no significant difference was observed among subgroups B2, $C 2$, and D2 $(p=0.126)$, while the change in $\gamma-\mathrm{H} 2 \mathrm{AX}$ MFI in subgroup A2 was found to be higher than those in the other three subgroups $\mathrm{B} 2, \mathrm{C} 2$, and $\mathrm{D} 2(p=0.001, p=$ 0.001 , and $p<0.001$, respectively).

\section{Objective and Subjective Image Quality Comparisons}

Supplementary Table 1 provides the results of objective image quality evaluations. The CT values in the $120-\mathrm{kVp}$ group were lower and the SNRs of the RCA, LAD, LCX, and aorta were significantly higher than those in the other three subgroups (all $p<0.001$ ) (Fig. 5). However, there were no significant differences in the CNRs of the RCA, LAD, LCX, and aorta among the 120-, 100-, and 80-kVp subgroups $(p=0.094, p=0.122, p=0.128$, and $p=0.143$, respectively). The image noise was the highest in the 70$\operatorname{kVp}$ group $(p=0.012)$.

As tube voltage decreased from $120 \mathrm{kVp}$ to $70 \mathrm{kVp}$, the scores for overall image quality decreased and those for noise increased significantly (all $p<0.001$ ). No study at any tube voltage was scored lower than 3 , but the ratio of score 5 for image quality and noise decreased as tube voltage decreased (Fig. 6). Interobserver agreement was good to fair for $120 \mathrm{kVp}(\kappa=0.704), 100 \mathrm{kVp}(\kappa=$ $0.647), 80 \mathrm{kVp}(\kappa=0.379)$, and $70 \mathrm{kVp}(\kappa=0.288)$. The interobserver agreements were significantly different among the four subgroups $(p<0.001)$.

\section{DISCUSSION}

This study found that DNA DSBs reached a plateau when weighed against image quality and radiation dose, as the tube voltage decreased from $120 \mathrm{kVp}$ to $100 \mathrm{kVp}$, which indicates that $100 \mathrm{kVp}$ may be optimal for CCTA studies on the basis of biological effects.

For many years, CCTA studies assessing radiation dosage focused on image quality for assessment of different radiation dose reduction methods. As reported, with IR techniques, a lower tube voltage or current would decrease the estimated effective radiation dose. Thus, the most effective method to reduce radiation dose, which involves the use of lower tube voltages such as $70 \mathrm{kVp}$, has been used for CCTA studies $(24,25)$. We have confirmed that excessive reductions in tube voltage harbor the risk of sacrificing image quality (CT values, SNR, and CNR), as reported in other studies $(24,26)$. However, the image quality of all included CCTA images still satisfied diagnostic requirements because the BMI of all included patients was set at less than $25 \mathrm{~kg} / \mathrm{m}^{2}$, and the dosage of contrast medium at the lower tube voltage was kept the same as 

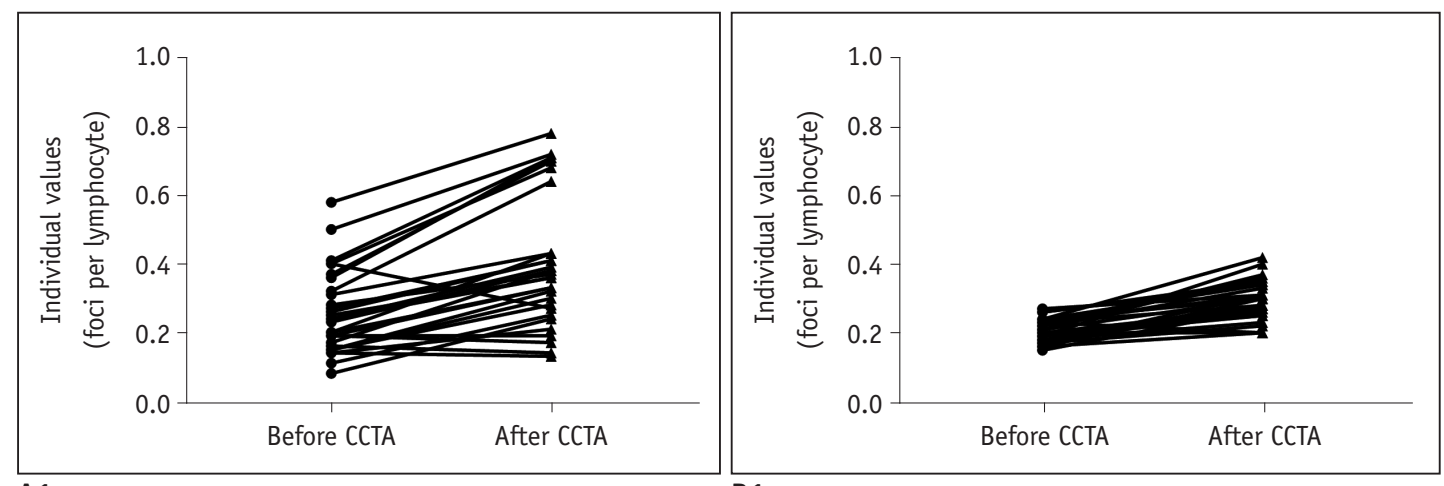

$$
\text { A1 }
$$

B1
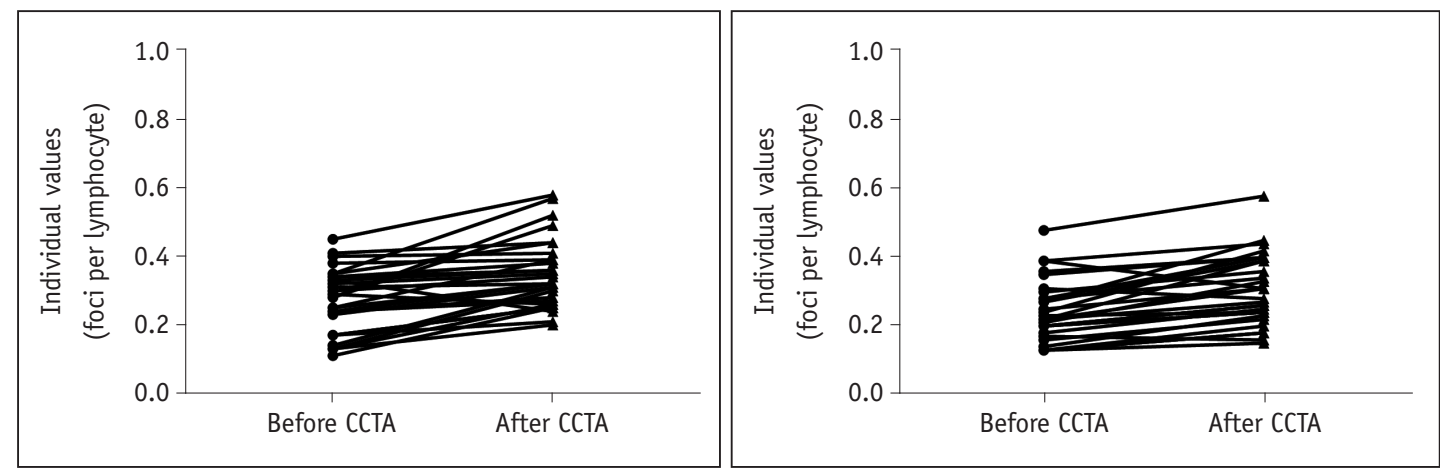

C1

D1
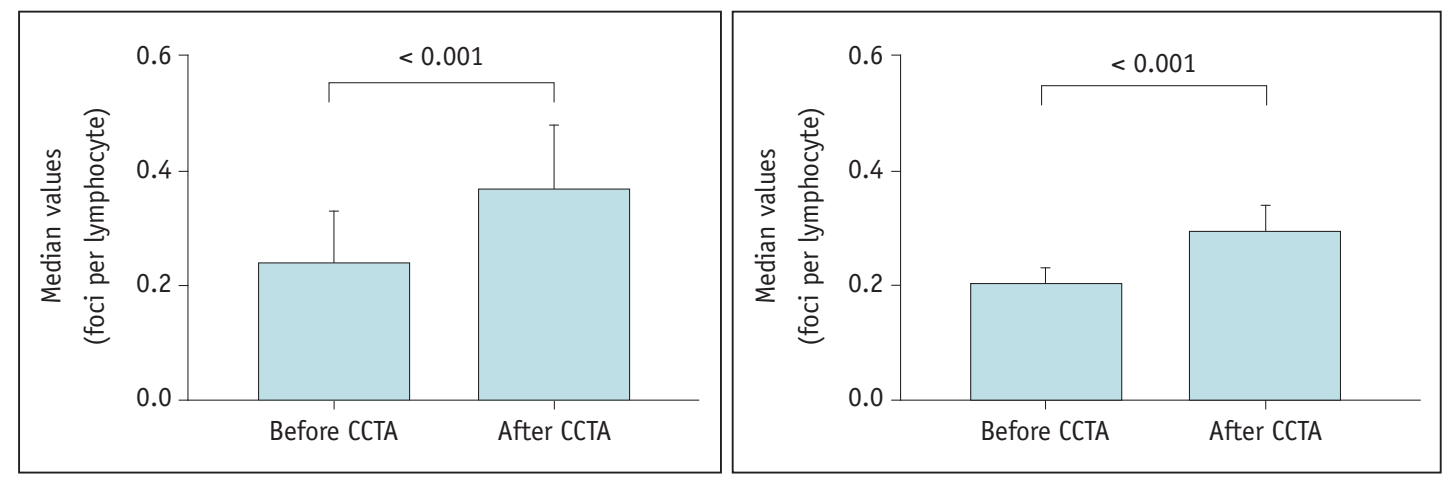

A2

B2
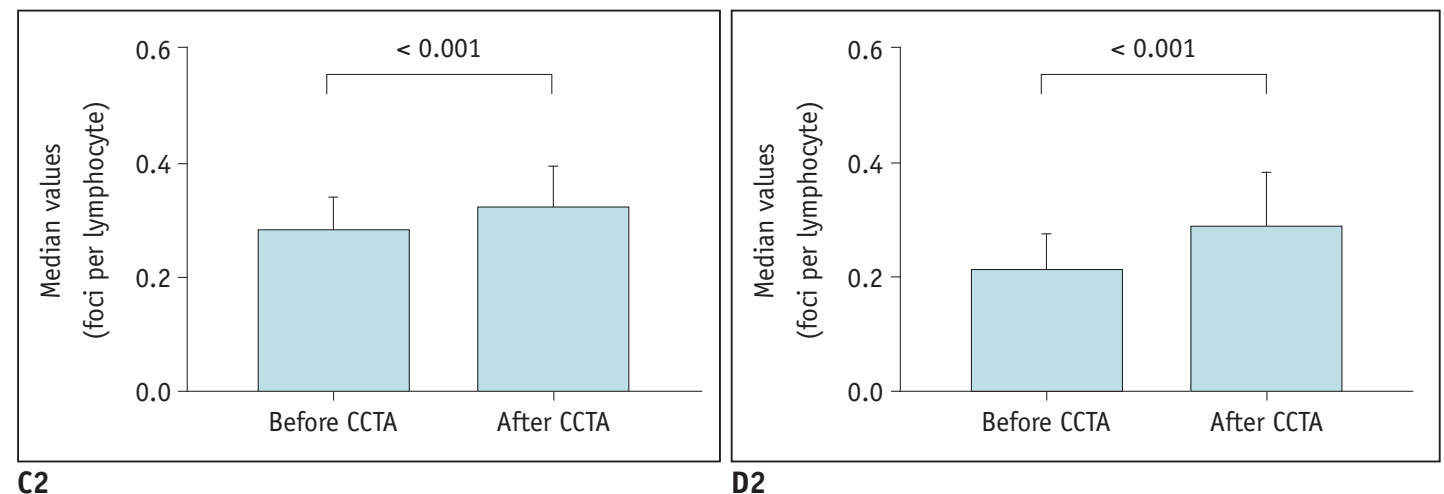

C2

Fig. 2. DNA DSBs before and after CCTA by immunofluorescence microscopy.

After CCTA, immunofluorescence microscopy shows significant increase in $\gamma$-H2AX foci per lymphocyte in subgroups examined with $120 \mathrm{kVp}$ (A1, A2), $100 \mathrm{kVp}$ (B1, B2), $80 \mathrm{kVp}(\mathbf{C 1}, \mathbf{C 2})$, and $70 \mathrm{kVp}$ (D1, D2). Bars indicate median values with median absolute deviation (A2-D2) and individual values are interconnected with line (A1-D1). CCTA = coronary CT angiography, DSB = double-strand break 


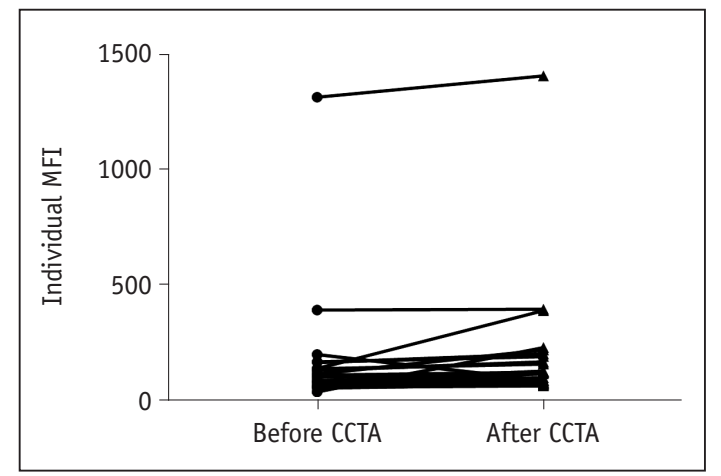

A1

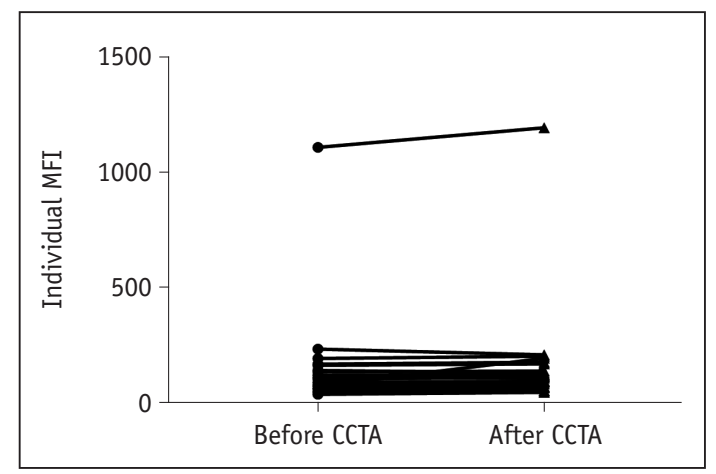

C1

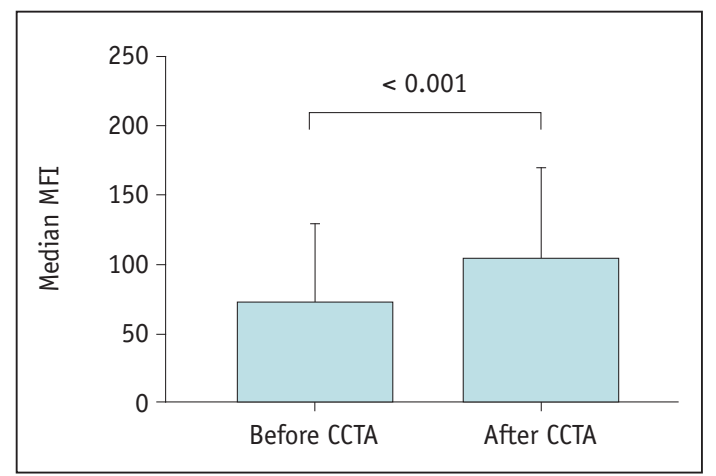

A2

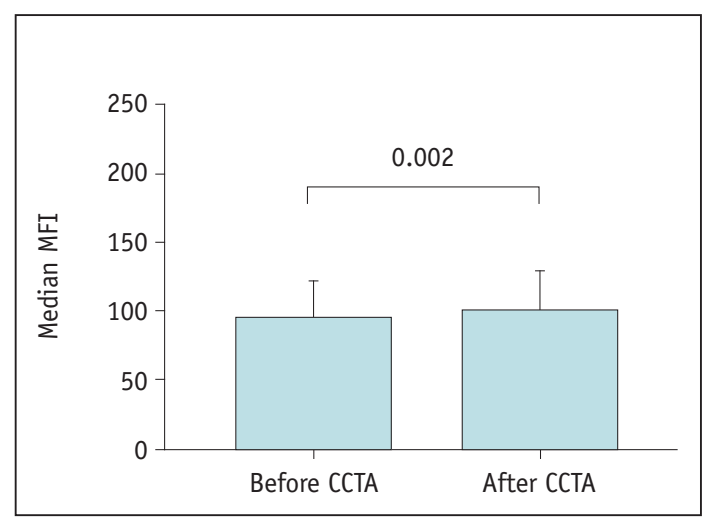

C2

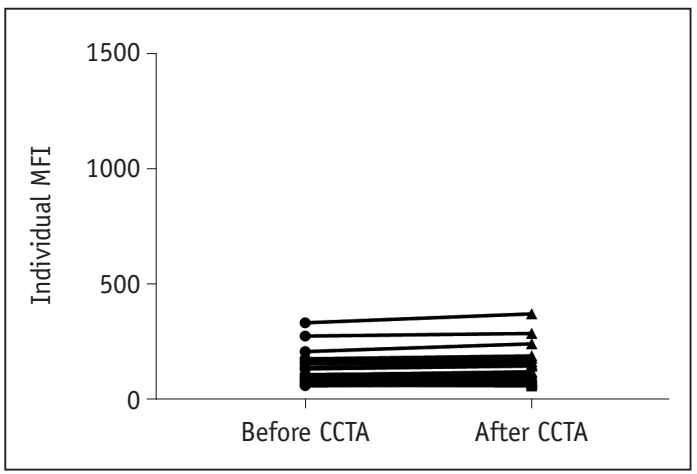

B1

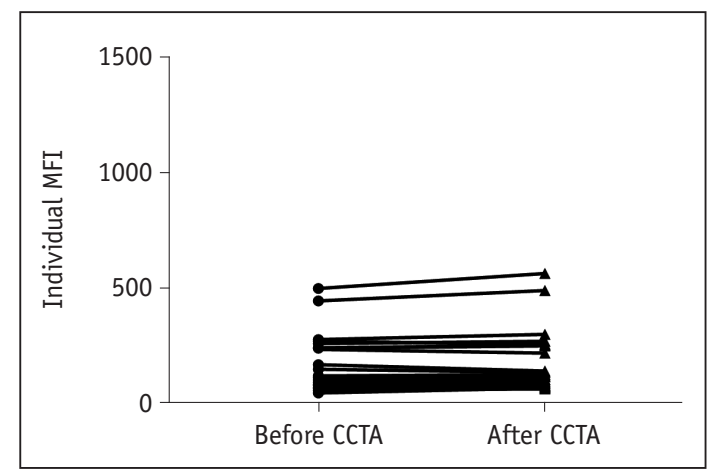

D1

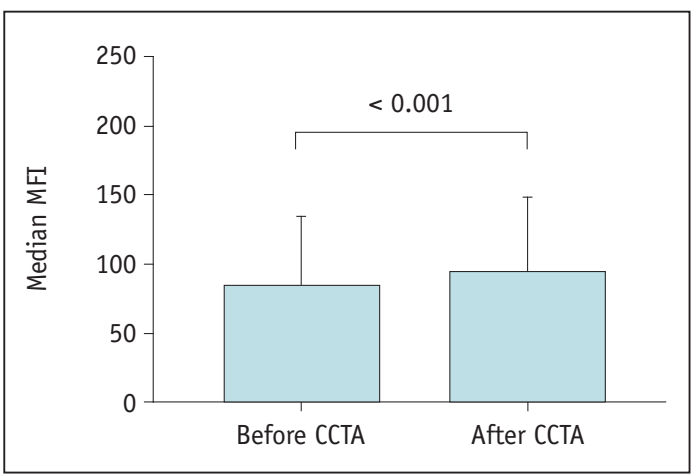

B2

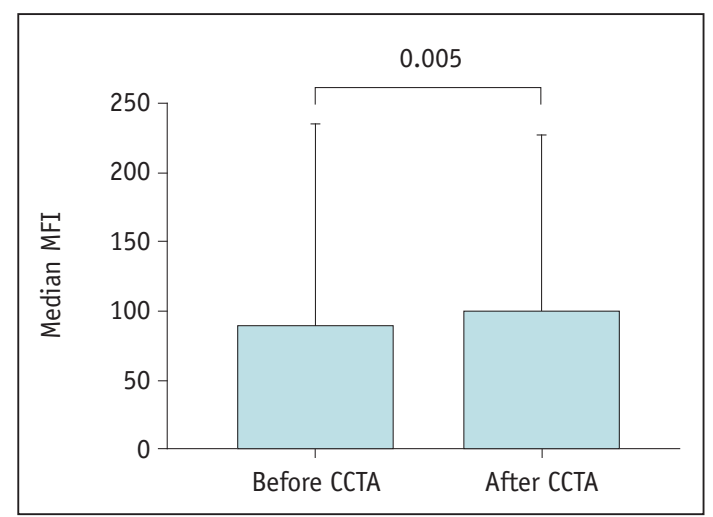

D2

Fig. 3. DNA DSBs before and after CCTA by flow cytometry.

Individual MFI and median MFI is increased significantly after CCTA (A2-D2) in subgroups examined with $120 \mathrm{kVp}$ (A1, A2), 100 kVp (B1, B2), $80 \mathrm{kVp}(\mathbf{C 1}, \mathbf{C 2})$, and $70 \mathrm{kVp}$ (D1, D2). Individual values are interconnected with line (A1-D1). MFI = mean fluorescence intensity 
that at higher tube voltages, which may have contributed to the overall diagnostic quality of CCTA acquisitions in this study.
The relationship between radiation dose and DNA damage has recently become a topic of intense research. The levels of DNA DSBs in peripheral blood lymphocytes are reported
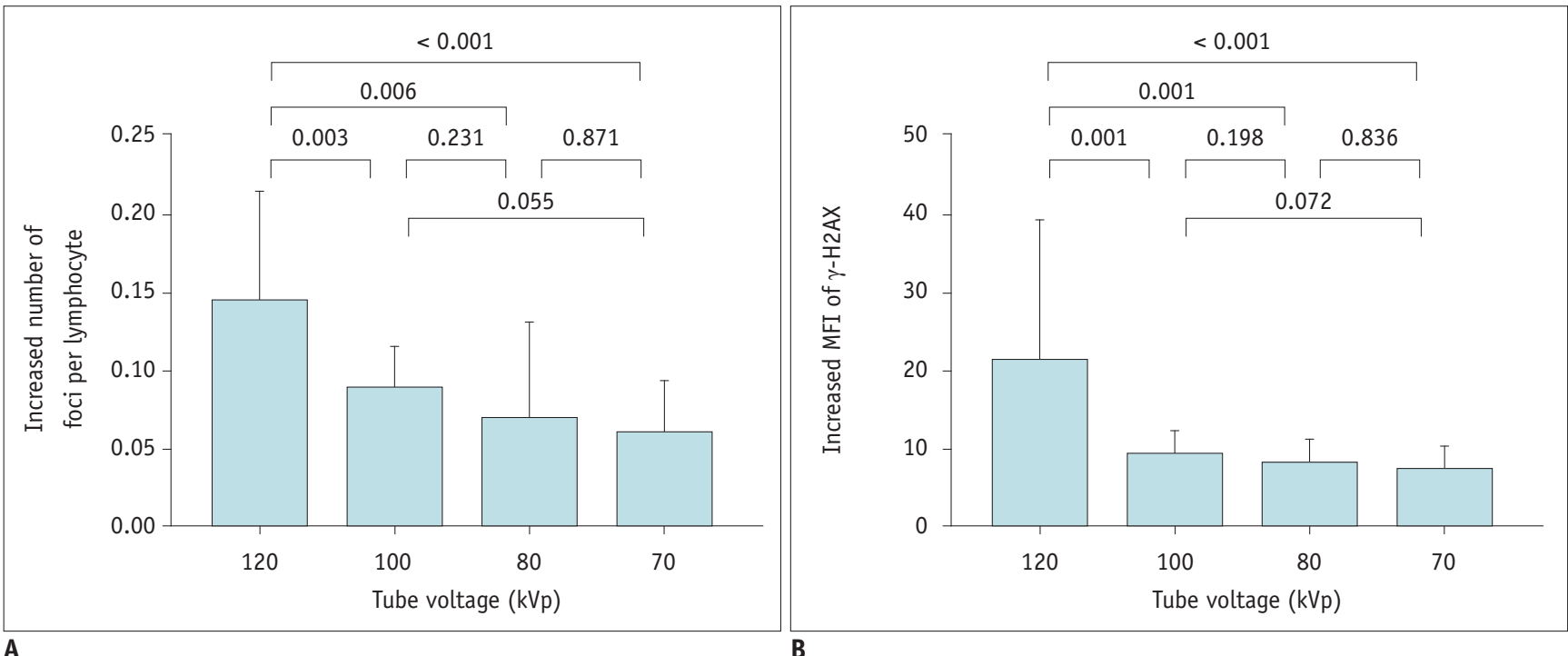

A

Fig. 4. Changes in $\gamma$-H2AX foci and MFI levels after CCTA examination.

A, B. Panels show changes in DNA DSBs as tube voltage decreased. There are no significant differences in DNA DSBs among 100-, 80-, and 70-kVp subgroups $(p=0.184,0.126)$. DNA DSB level at $120 \mathrm{kVp}$ is higher than those in other three subgroups (all $p<0.05)$ are.

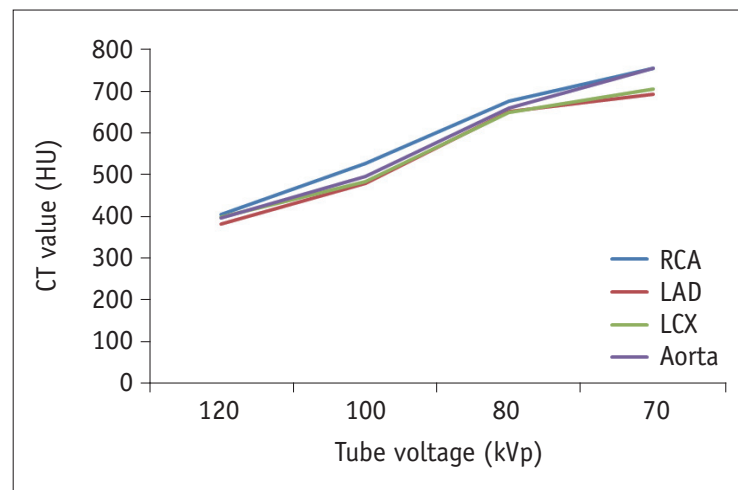

A

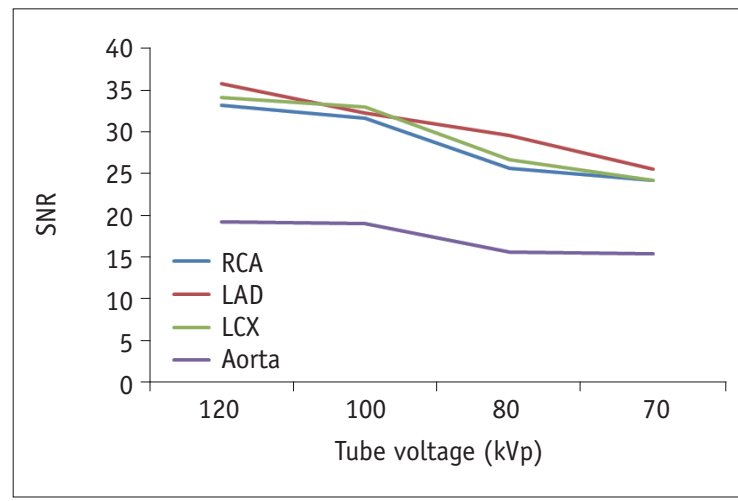

C

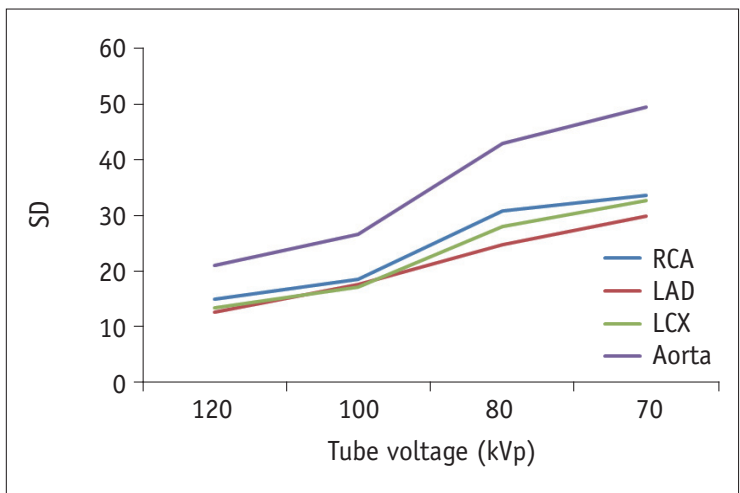

B

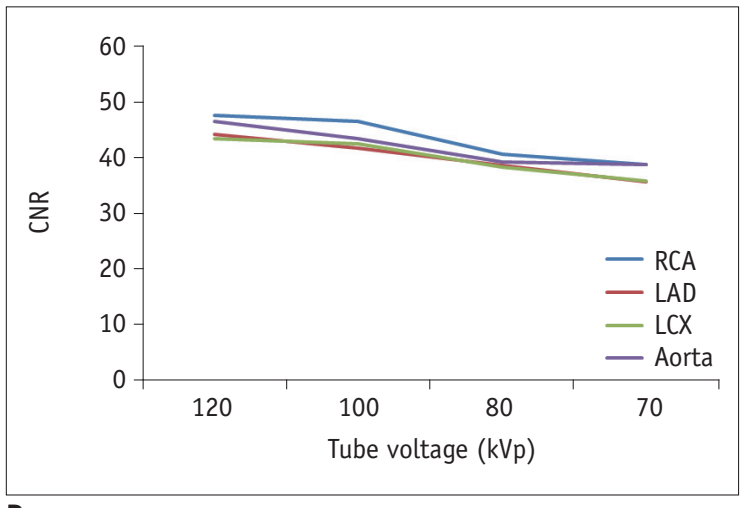

Fig. 5. Objective image quality in different tube voltage groups.

CT values (A) and noise (B) of RCA, LAD, LCX, and aorta increased gradually when tube voltage decreased, while SNR (C) and CNR (D) decreased. $\mathrm{CNR}=$ contrast-to-noise ratio, $\mathrm{LAD}=$ left anterior descending artery, $\mathrm{LCX}=$ left circumflex artery, $\mathrm{RCA}=$ right coronary artery, $\mathrm{SD}=$ standard deviation, SNR = signal-to-noise ratio 


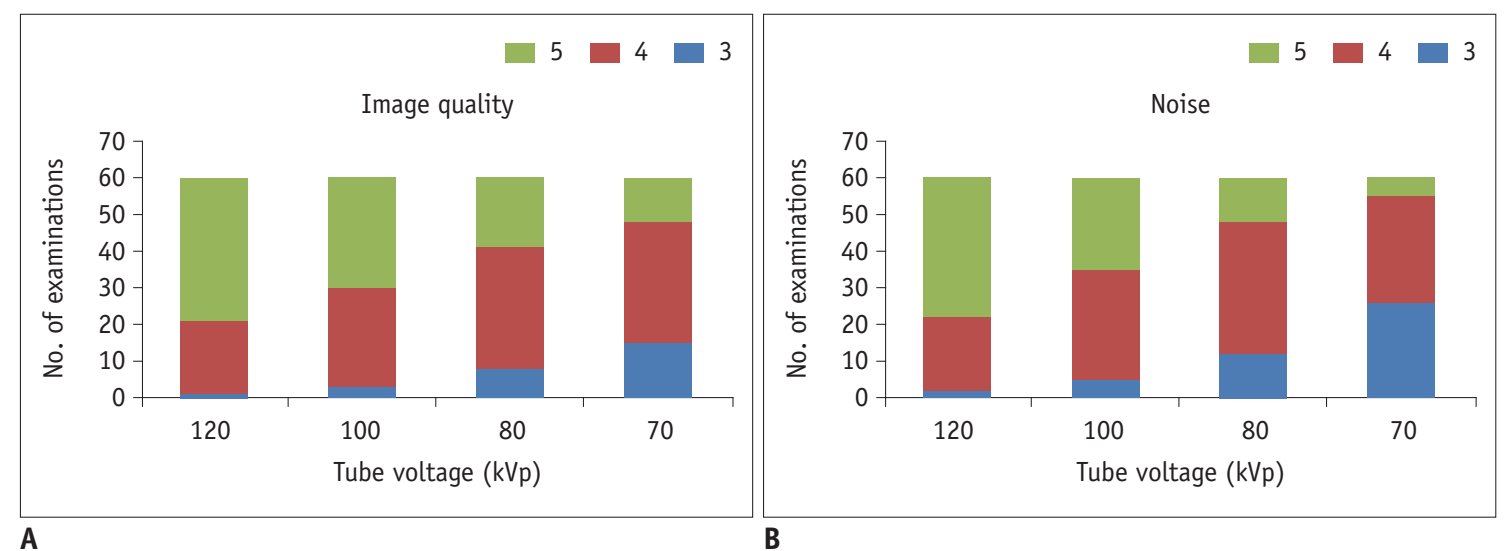

Fig. 6. Image quality and noise distribution in different tube voltage groups. No CCTA study at any tube voltage group scored less than 3 points in image quality (A) and noise (B). However, ratio of 5-point examinations increased as tube voltage increased in both of image quality and noise.

to be related to the radiation dose $(27,28)$. Zhang et al. (21) reported that a lower tube voltage $(80 \mathrm{kVp})$ caused more severe DNA DSBs than a higher tube voltage (140 kVp), which suggested that reducing tube voltage may actually be harmful because of the increased scattered radiation absorbed by the body. However, in the current study, the increase in the levels of DNA DSBs after CCTA at 100, 80, and $70 \mathrm{kVp}$ showed no statistical difference, a finding supported by the results reported by Brand et al. (29). There was a significant decline in the estimated radiation dose and image quality as the tube voltage decreased, but DNA DSB levels remained stable although tube voltage decreased from $100 \mathrm{kVp}$ to $70 \mathrm{kVp}$. These results suggest that the effect of lower radiation doses (< $5 \mathrm{mSv}$ ) on DNA DSBs was very minimal. Thus, a biological effect-based approach may be more relevant in weighing ionizing radiation damage and image quality than an estimated radiation dose strategy.

In our study, we used both immunofluorescence staining and flow cytometry to assess the relationships among DNA damage, estimated radiation dose, and image quality (19). The results showed that when tube voltage was reduced from $120 \mathrm{kVp}$ to $100 \mathrm{kVp}$, the DNA DSB level reduced by $30 \%$, which was previously reported by immunofluorescence microscopy (20) and was also supported by a similar reduction observed by immunofluorescence (39\%). Although immunofluorescence microscopy has been reported to show much higher sensitivity, flow cytometry had sufficient analytical sensitivity to measure the levels of DNA damage due to the large number of cells analyzed in clinical practice, as noted by Johansson et al. (18). Therefore, both methods could be used based on the laboratory conditions.

We acknowledge certain limitations with this study. First, the sample size in the subgroups was small, and a larger population will be needed in future studies to validate our findings further. Second, although we used two methods to demonstrate the same results for the quantification of DNA DSBs, they were not used simultaneously in the same patient population. Additionally, other detection methods could be used for DNA DSBs in future studies. Third, the effect of the contrast medium on DNA DSBs was not investigated because this was not a variable designated to be optimized with respect to tube voltage at the outset of the study. Fourth, different radiation doses resulting from different tube voltages may affect our observed results. Except tube voltage, all remaining scan parameters were kept constant in our study. Thus, the interaction of tube voltage and radiation dose on DNA DSBs needs to be studied further. Finally, we did not compare the technique used herein with digital subtraction angiography or other gold standards. However, Lee et al. (30) have shown that tube voltage does not affect the accuracy of stenosis measurement based on a phantom study.

In conclusion, this study indicates that a biological effect-based approach is rational to weigh radiation damage against image quality. A 100-kVp tube voltage may be optimal for CCTA studies if DNA DSBs are weighted, rather than estimated effective radiation dose, vis-a-vis image quality.

\section{Supplementary Materials}

The Data Supplement is available with this article at https://doi.org/10.3348/kjr.2019.0932. 


\section{Conflicts of Interest}

UJS is a consultant for and/or receives research support from Astellas, Bayer, Elucid BioImaging, General Electric, Guerbet, HeartFlow, and Siemens Healthineers. The other authors have no conflicts of interest to disclose.

\section{ORCID iDs}

Long Jiang Zhang

https://orcid.org/0000-0002-6664-7224

Zhu Xiao Lin

https://orcid.org/0000-0003-0119-2721

Fan Zhou

https://orcid.org/0000-0003-4837-5955

U. Joseph Schoepf

https://orcid.org/0000-0002-6164-5641

Balakrishnan Pillai

https://orcid.org/0000-0001-8768-1221

Chang Sheng Zhou

https://orcid.org/0000-0001-5306-2801

Wei Quan

https://orcid.org/0000-0002-2077-9213

Xue Qin Bao

https://orcid.org/0000-0002-0866-7104

Guang Ming Lu

https://orcid.org/0000-0003-4913-2314

\section{REFERENCES}

1. Mark DB, Berman DS, Budoff MJ, Carr JJ, Gerber TC, Hecht HS, et al. ACCF/ACR/AHA/NASCI/SAIP/SCAI/SCCT 2010 expert consensus document on coronary computed tomographic angiography: a report of the American College of Cardiology Foundation Task Force on Expert Consensus Documents. J Am Coll Cardiol 2010;55:2663-2699

2. Bloomfield GS, Gillam LD, Hahn RT, Kapadia S, Leipsic J, Lerakis $\mathrm{S}$, et al. A practical guide to multimodality imaging of transcatheter aortic valve replacement. JACC CardiovasC Imaging 2012;5:441-455

3. Martinez MW, Kirsch J, Williamson EE, Syed IS, Feng D, Ommen $\mathrm{S}$, et al. Utility of nongated multidetector computed tomography for detection of left atrial thrombus in patients undergoing catheter ablation of atrial fibrillation. JACC Cardiovasc Imaging 2009;2:69-76

4. Caruso D, De Santis D, Biondi T, Panvini N, Zerunian M, Rivosecchi $F$, et al. Half-dose coronary artery calcium scoring: impact of iterative reconstruction. J Thorac Imaging 2019;34:18-25

5. Tabari A, Lo Gullo R, Murugan V, Otrakji A, Digumarthy $S$, Kalra M. Recent advances in computed tomographic technology: cardiopulmonary imaging applications. J Thorac
Imaging 2017;32:89-100

6. Pontone G, Muscogiuri G, Baggiano A, Andreini D, Guaricci AI, Guglielmo $M$, et al. Image quality, overall evaluability, and effective radiation dose of coronary computed tomography angiography with prospective electrocardiographic triggering plus intracycle motion correction algorithm in patients with a heart rate over 65 beats per minute. J Thorac Imaging 2018;33:225-231

7. Zhang LJ, Qi L, Wang J, Tang CX, Zhou CS, Ji XM, et al. Feasibility of prospectively ECG-triggered high-pitch coronary CT angiography with $30 \mathrm{~mL}$ iodinated contrast agent at 70 kVp: initial experience. Eur Radiol 2014;24:1537-1546

8. Spearman JV, Schoepf UJ, Rottenkolber M, Driesser I, Canstein C, Thierfelder KM, et al. Effect of automated attenuation-based tube voltage selection on radiation dose at CT: an observational study on a global scale. Radiology 2016;279:167-174

9. Mangold S, Wichmann JL, Schoepf UJ, Poole ZB, Canstein C, Varga-Szemes A, et al. Automated tube voltage selection for radiation dose and contrast medium reduction at coronary $\mathrm{CT}$ angiography using $3(\mathrm{rd})$ generation dual-source CT. Eur Radiol 2016;26:3608-3616

10. Zhang LJ, Wang Y, Schoepf UJ, Meinel FG, Bayer RR 2nd, Qi L, et al. Image quality, radiation dose, and diagnostic accuracy of prospectively ECG-triggered high-pitch coronary CT angiography at $70 \mathrm{kVp}$ in a clinical setting: comparison with invasive coronary angiography. Eur Radiol 2016;26:797-806

11. Kuefner MA, Brand M, Ehrlich J, Braga L, Uder M, Semelka RC. Effect of antioxidants on X-ray-induced $\gamma-\mathrm{H} 2 \mathrm{AX}$ foci in human blood lymphocytes: preliminary observations. Radiology 2012;264:59-67

12. Cadet J, Douki T, Ravanat JL. Oxidatively generated base damage to cellular DNA. Free Radic Biol Med 2010;49:9-21

13. Corre I, Niaudet C, Paris F. Plasma membrane signaling induced by ionizing radiation. Mutat Res 2010;704:61-67

14. Haro KJ, Sheth A, Scheinberg DA. Dysregulation of IRP1mediated iron metabolism causes gamma ray-specific radioresistance in leukemia cells. PLoS One 2012;7:e48841

15. de González AB, Darby S. Risk of cancer from diagnostic $\mathrm{X}$-rays: estimates for the UK and 14 other countries. Lancet 2004;363:345-351

16. Fiechter M, Stehli J, Fuchs TA, Dougoud S, Gaemperli 0 , Kaufmann PA. Impact of cardiac magnetic resonance imaging on human lymphocyte DNA integrity. Eur Heart J 2013;34:2340-2345

17. Muslimovic A, Ismail IH, Gao Y, Hammarsten 0. An optimized method for measurement of gamma-H2AX in blood mononuclear and cultured cells. Nat Protoc 2008;3:1187-1193

18. Johansson P, Fasth A, Ek T, Hammarsten 0 . Validation of a flow cytometry-based detection of $\gamma-\mathrm{H} 2 \mathrm{AX}$, to measure DNA damage for clinical applications. Cytometry B Clin Cytom 2017;92:534-540

19. Nguyen PK, Lee WH, Li YF, Hong WX, Hu S, Chan C, et al. Assessment of the radiation effects of cardiac CT angiography 
using protein and genetic biomarkers. JACC CardiovasC Imaging 2015;8:873-884

20. Kuefner MA, Grudzenski S, Hamann J, Achenbach S, Lell $M$, Anders K, et al. Effect of CT scan protocols on x-rayinduced DNA double-strand breaks in blood lymphocytes of patients undergoing coronary CT angiography. Eur Radiol 2010;20:2917-2924

21. Zhang B, Gong JP, Zhang W, Pan SW. Influence of tube voltage in CT-induced DNA double-strand breaks in human peripheral blood lymphocytes. Chin J Nucl Med Mol Imaging 2016;36:466-467

22. Halliburton SS, Abbara S, Chen MY, Gentry R, Mahesh M, Raff GL, et al. SCCT guidelines on radiation dose and doseoptimization strategies in cardiovascular CT. J CardiovasC Comput Tomogr 2011;5:198-224

23. Wu Q, Wang $Y$, Kai H, Wang T, Tang $X$, Wang $X$, et al. Application of $80-k V p$ tube voltage, low-concentration contrast agent and iterative reconstruction in coronary CT angiography: evaluation of image quality and radiation dose. Int J Clin Pract 2016;70 Suppl 9B:B50-B55

24. Zhang LJ, Qi L, De Cecco CN, Zhou CS, Spearman JV, Schoepf $\mathrm{UJ}$, et al. High-pitch coronary CT angiography at $70 \mathrm{kVp}$ with low contrast medium volume: comparison of 80 and $100 \mathrm{kVp}$ high-pitch protocols. Medicine (Baltimore) 2014;93:e92

25. Wang W, Zhao YE, Qi L, Li X, Zhou CS, Zhang LJ, et al. Prospectively ECG-triggered high-pitch coronary CT angiography at $70 \mathrm{kVp}$ with $30 \mathrm{~mL}$ contrast agent: an intraindividual comparison with sequential scanning at 120 kVp with 60mL contrast agent. Eur J Radiol 2017;90:97-105

26. Ebersberger U, Tricarico F, Schoepf UJ, Blanke P, Spears JR, Rowe GW, et al. CT evaluation of coronary artery stents with iterative image reconstruction: improvements in image quality and potential for radiation dose reduction. Eur Radiol 2013;23:125-132

27. Beels L, Bacher K, Smeets P, Verstraete K, Vral A, Thierens $H$. Dose-length product of scanners correlates with DNA damage in patients undergoing contrast CT. Eur J Radiol 2012;81:1495-1499

28. Löbrich M, Rief N, Kühne M, Heckmann M, Fleckenstein J, Rübe $C$, et al. In vivo formation and repair of DNA doublestrand breaks after computed tomography examinations. Proc Natl Acad Sci U S A 2005;102:8984-8989

29. Brand M, Sommer M, Achenbach S, Anders K, Lell M, Löbrich $M$, et al. X-ray induced DNA double-strand breaks in coronary CT angiography: comparison of sequential, low-pitch helical and high-pitch helical data acquisition. Eur J Radiol 2012;81:e357-e362

30. Lee SM, Lee W, Chung JW, Park EA, Park JH. Effect of kVp on image quality and accuracy in coronary CT angiography according to patient body size: a phantom study. Int $\mathrm{J}$ Cardiovasc Imaging 2013;29 Suppl 2:83-91 